\title{
Evaluación de un protocolo para valorar situaciones de desprotección infantil: la opinión de los técnicos*
}

\author{
Evaluation of a protocol to assess situations where a child is in need \\ of protection from the child protection workers' point of view
}

Recibido: marzo 3 de 2010 | Revisado: agosto 15 de 2010 | Aceptado: septiembre 17 de 2010

\author{
EDUARDO MARTÍN ${ }^{* *}$ \\ RAMÓn ACIEGO DE MENDOZA \\ Universidad de La Laguna, Tenerife, España
}

SICI: 1657-9267(201112) 10:3<693:EPVSDI >2.3.TX;2-P

Para citar este artículo. Martín, E. \& Aciego de Mendoza, R. (2011). Evaluación de un protocolo para valorar situaciones de desprotección infantil: la opinión de los técnicos. Universitas Psychologica, 10 (3), 693-704.

* Este trabajo se ha elaborado a través de un contrato de $\mathrm{I}+\mathrm{D}+\mathrm{i}$ financiado por el Instituto Insular de Atención Social y Sociosanitaria (IASS) de Tenerife, gestionado por la Fundación Canaria Empresa Universidad de La Laguna (FCEULL).

** Facultad de Psicología, Universidad de La Laguna. Campus de Guajara s/n, 38205. La Laguna, España. E-mails: edmartin@ull.es, raciego@ull.es
RESUMEN

El objetivo de este trabajo es conocer la valoración que los técnicos municipales hacen de un protocolo cuyo objetivo es unificar, en los diferentes municipios de la isla de Tenerife, el procedimiento de valoración de los casos de desprotección infantil. 126 técnicos respondieron a un cuestionario en el que se les pedía su opinión sobre tres aspectos diferentes: el contenido del protocolo, los efectos de su aplicación y los recursos que facilitan su implementación. Los resultados indican que los profesionales valoran positivamente el contenido y los efectos del protocolo, sobre todo los referentes a las condiciones en las que realizan su labor, la unificación de criterios y la coordinación horizontal. Los recursos más valorados en la implementación del protocolo son el acompañamiento de técnicos especializados durante el proceso de implementación, la existencia de espacios de encuentro y una buena dinámica de trabajo en equipo. Se encontraron algunas diferencias en la valoración, en función del perfil profesional. Se concluye que es adecuada la creación de este tipo de instrumentos, a través de procesos participativos, y que su implementación se ve mediatizada por algunas variables ajenas al instrumento.

Palabras clave autores

Protocolo de valoración, protección infantil, equipos multidisciplinares, servicios

sociales municipales, estudio descriptivo mediante encuesta.

Palabras clave descriptores

Pruebas psicológicas, diseño exploratorio, investigación cuantitativa.

\begin{abstract}
A B S T R AC T
The aim of this study is to find out council child protection officers' assessment of a protocol that unifies the different evaluation procedures in district councils on the island of Tenerife in cases where a child is in need of protection. 126 child protection officers answered an ad hoc questionnaire in which their opinions on three aspects were requested: the content of the protocol, the effects of its application and the resources to facilitate its implementation. Results indicate that professionals value positively the content and effects of the protocol, above all in regard to the conditions in which they carry out their work, the unification of criteria and horizontal co-ordination. The resources most valued in the implementation of the protocol are: being accompanied by specialists during the implementation processes; the existence of opportunities for meetings and a good teamwork dynamic. There were some differences in the assessment of the professional's profile. In conclusion, the creation of this type of instrument through parti-
\end{abstract}


cipative processes and its implementation when influenced by some variables not directly connected to the instrument is considered appropriate.

Key words authors

Assessment protocol, child protection, multidisciplinary teams, council social services, descriptive design.

Key words plus

Psychological test, descriptive design, quantitative research.

El maltrato infantil es un grave problema social que tiene secuelas en el colectivo más vulnerable de la sociedad como es el de la infancia. Se trata además de un problema en el que es difícil obtener datos epidemiológicos fiables, debido a los diferentes criterios utilizados por las distintas agencias y administraciones a la hora de identificar los distintos tipos de maltrato infantil (Fallon et al., 2010). En España, este problema se complica aún más con la descentralización y la transferencia de responsabilidades, en materia de protección infantil, a las diferentes comunidades autónomas, en la década de los 80 del siglo pasado. Actualmente, se ha comprobado que el desarrollo, tanto reglamentario como de dotación de recursos, del sistema de protección infantil ha sido diferente en cada comunidad autónoma (González, 2002), observándose grandes diferencias entre ellas (De Paúl, 2009). No obstante, en la mayoría de los casos se le asigna a los servicios sociales municipales la responsabilidad de investigar las posibles situaciones de desprotección infantil que se notifican (Arruabarrena, 2009). El motivo fundamental de esta descentralización es que los recursos más cercanos a los ciudadanos son los servicios sociales municipales y, por lo tanto, tienen un acceso más fácil y más rápido a los casos que son notificados.

No es objetivo de este trabajo analizar en profundidad las bases y el funcionamiento del sistema de protección infantil en España, para lo que remitimos a la lectura del número monográfico publicado recientemente en la revista Papeles del Psicólogo (Consejo General de Colegios Oficiales de Psicólogos, 2009).

Los esfuerzos de la investigación en este campo se han centrado tanto en la clasificación de las diferentes situaciones de desprotección infantil (Arruabarrena \& De Paúl, 1994; De Paúl, 2002;
English, Bangdiwala \& Runyan, 2005; Fallon et al., 2010; Manly, 2005; Muela, 2008) y de las necesidades de la infancia (López, 1995, 2008; López, Torres, Fuertes, Sánchez \& Merino, 1995), como en la elaboración de procedimientos y criterios de investigación e intervención (Arruabarrena, 2002, 2009; Darlington, Healy \& Feeney, 2010; Observatorio de la Infancia, 2008; Rodrigo \& Máiquez, 2007). Además, la aparición del concepto de resiliencia ha permitido introducir los factores de protección como factores complementarios a los de riesgo en la investigación, aportando pautas y criterios para la intervención posterior (Daniel, Vincent, Farrall \& Arney, 2009; Lázaro, 2009; Rodrigo, Camacho, Máiquez, Byrne \& Benito, 2009; Rodrigo, Máiquez, Martín \& Byrne, 2008).

Las decisiones que los profesionales toman a la hora de investigar situaciones de desprotección infantil, pueden tener unas repercusiones muy importantes, tanto para los menores como para las familias, y no siempre se pueden tomar con base en criterios y pruebas claras. Aunque se propone que en la investigación se combinen procedimientos cuantitativos para medir el riesgo con los juicios clínicos de los técnicos (Shlonsky \& Wagner, 2005), lo cierto es que en muchas ocasiones existen sesgos y se toman decisiones con base en criterios subjetivos, al tratarse de un proceso complejo en el que no siempre es posible acceder a la información y que se realiza, en muchas ocasiones, a contrarreloj (Darlington et al., 2010). En este sentido, Runyan et al. (2005) encontraron que un $10 \%$ de los casos de maltrato físico y abuso sexual eran clasificados por los técnicos como casos de negligencia. Otros trabajos (Arad-Davidzon \& Benbenishty, 2008; Britner \& Mossler, 2002) han demostrado que, ante una misma situación, algunos técnicos son más propensos a tomar decisiones que posibiliten la permanencia de los menores en la familia, mientras que otros tienen una mayor tendencia a tomar decisiones que conllevan la separación del menor de su familia. Jent et al. (2009) encontraron que los profesionales encargados de realizar la investigación tienden a darle un mayor peso a los factores de riesgo que a las fortalezas y factores protectores presentes en el caso, a la hora de tomar decisiones. 
En nuestro país, resultados similares encontraron Rodríguez, Camacho, Rodrigo, Martín y Máiquez (2006) al comprobar que, en ciertos casos, los técnicos municipales tienden a sobreestimar los factores de riesgo en sus valoraciones, posiblemente debido a que el principio en el que se basa la legislación es la protección y el interés último del menor. Algunas voces (Martín, 2005, 2009) vienen advirtiendo que los valores que subyacen al sistema de protección facilitan la estigmatización de las familias, lo que explicaría, al menos en parte, los sesgos aquí expuestos.

Todo lo comentado anteriormente justifica la necesidad de contar con procedimientos de investigación consensuados claros, que utilicen un lenguaje común, que faciliten la coordinación entre profesionales y administraciones, tanto horizontal como verticalmente (De Paúl, 2009), y que promuevan el trabajo en equipo, lo que permitiría afrontar mejor la complejidad de los casos y reducir los sesgos individuales (Rodrigo et al., 2008). Además, es conveniente contar con la opinión de los propios profesionales que realizan las labores de investigación, ya que nos pueden aportar una información valiosa sobre las dificultades con las que se encuentran al desarrollar su trabajo (Darlington et al., 2010). Son bastante escasas las investigaciones que tienen en cuenta la opinión de los profesionales. En nuestro país, Casas y Montserrat (2002) estudiaron la puesta en marcha de los equipos interdisciplinares en Cataluña, y encontraron que los profesionales demandaban un mayor apoyo técnico y formación permanente. Además, en sintonía con lo comentado anteriormente, comprobaron que las decisiones se tomaban con criterios diferentes, ya que la experiencia no les había llevado a generar un cuerpo de conocimiento común.

La opinión de los profesionales también es importante a la hora de valorar las herramientas e instrumentos que se usan en la práctica diaria. Un concepto relevante en este sentido es el de metaprograma. Hernández (1993) plantea que la eficacia de cualquier instrumento o programa no depende solo de su propia bondad interna, sino que existen otras variables metaprograma que van a mediatizar el éxito en su implementación.
Así, aspectos como el grado en el que los profesionales que lo van a aplicar asumen la teoría y las directrices del programa, el dominio que tengan de los contenidos y los procedimientos a desarrollar, las expectativas positivas generadas hacia el programa, y la percepción de la propia capacidad para desarrollarlo, estarían detrás del éxito en la implementación y puesta en marcha de cualquier cambio o reforma en la práctica profesional. Por esto, parece necesario conocer la valoración que los profesionales hacen de cualquier herramienta de trabajo.

Es en este contexto en el que se ubica este estudio que pretende evaluar, desde el punto de vista de los profesionales, un protocolo unificado para valorar las situaciones de desprotección infantil en la isla de Tenerife. En la Comunidad Autónoma Canaria, la ley 1/97 de atención integral a los menores establece que las administraciones locales son las encargadas de investigar, a través de los equipos multidisciplinares formados por psicólogos, trabajadores sociales y educadores, los posibles casos de desprotección notificados, mientras que las administraciones insulares tienen, entre sus funciones, la de prestar apoyo y asesoramiento especializado. En este marco legislativo, el Instituto Insular de Atención Social y Sociosanitaria (IASS), en coordinación con los 31 municipios de la isla, impulsó la elaboración de un protocolo unificado para valorar las situaciones de desprotección infantil en la isla.

Concretamente, este trabajo tiene los siguientes objetivos. En primer lugar, conocer la valoración que los técnicos municipales hacen del contenido y las herramientas del protocolo. En segundo lugar, analizar cuáles son los efectos de la aplicación del protocolo desde el punto de vista de los profesionales. Y en tercer y último lugar, conocer cuáles son los factores que facilitan la implementación y la efectividad del protocolo.

De acuerdo con los objetivos planteados se ha optado por realizar un estudio descriptivo mediante encuesta (Montero \& León, 2007). Para la elaboración del artículo se han seguido los criterios propuestos por Ramos-Álvarez, Moreno-Fernández, Valdés-Conroy y Catena (2008). 


\section{Método}

\section{Participantes}

En este trabajo han participado los 126 técnicos de los servicios sociales municipales de 29 de los 31 municipios de la isla de Tenerife que habían utilizado el protocolo. En los dos municipios que no participaron ningún técnico municipal había aplicado el protocolo en el momento de recoger la información, por lo que la tasa de respuesta fue del $100 \%$ sobre los técnicos municipales que lo habían utilizado. En cuanto al perfil profesional, 32 son psicólogos (25.4\%), 60 trabajadores sociales $(47.6 \%)$ y 34 educadores ( $27 \%$ ). De ellos, 66 (52.4\%) habían participado en la elaboración del protocolo y 60 (47.6\%) no.

\section{Instrumentos}

Protocolo de valoración de situaciones de desprotección infantil en la isla de Tenerife (Instituto Insular de Atención Social y Sociosanitaria, 2008)

Este instrumento se elaboró con el objetivo fundamental de unificar el proceso de investigación de casos de desprotección infantil que se realiza en los 31 municipios de la isla de Tenerife. El protocolo se compone de los contenidos teóricos y procedimentales necesarios para que los profesionales realicen de manera adecuada su labor. Se pretende que en todos los casos se tengan en cuenta los mismos criterios, se utilice el mismo lenguaje y se den los mismos pasos, así como que se utilicen los mismos modelos de registro y de informes en los diferentes municipios. El protocolo plantea el proceso de investigación en dos fases (Arruabarrena, 2009): investigación previa (IP) y valoración especializada. La fase de IP se inicia en el momento en el que se recibe una notificación en los SS municipales, y tiene como objetivos investigar si se está produciendo una posible situación de desprotección infantil, atender a las necesidades urgentes de los menores y las familias si las hubiera, y decidir si se continúa con un proceso de valoración. Quien diseña y realiza el trabajo de investigación en esta fase es el/la trabajador/a social, aunque la valoración final se realiza en equipo.

Si en esta fase de IP se decide iniciar una segunda fase de valoración especializada, esta se realizará para cubrir los siguientes objetivos: determinar las causas de la posible situación de desprotección, determinar las secuelas que dicha situación ha provocado al menor o menores implicados, determinar cuáles son los factores de riesgo y de protección presentes, realizar un pronóstico del caso, y, por último, definir las necesidades del menor y la familia y las propuestas de intervención. Esta fase se realiza en equipo, por lo que el primer paso es realizar una planificación conjunta del proceso, para lo cual el protocolo propone el uso de una planilla de planificación conjunta, mediante la que, antes de iniciar el proceso de valoración especializada, se decida qué fuentes se van a consultar y qué técnico se encargará de ellas, qué información se va a recabar de cada fuente y qué métodos se van a utilizar para recoger la información. Una vez realizadas las valoraciones especializadas, se integran sus resultados en un informe, en función de cuatro aspectos: la evaluación del estado del/la menor, la evaluación del riesgo, el pronóstico del caso y las necesidades de intervención. Todo el proceso se puede visualizar en la Figura 1.

\section{Cuestionario de evaluación para profesionales}

Se trata de un cuestionario ad hoc diseñado para conocer cómo valoran los técnicos municipales el protocolo. Para este trabajo se utilizan solo tres de sus apartados. En el primero de ellos, se recoge la valoración del contenido teórico (conceptos, criterios y procedimientos) y los instrumentos (modelos de registro y de informe). Se les pide a los técnicos que los valoren en una escala de 1 (Muy inadecuado), 2 (Inadecuado), 3 (Adecuado) y 4 (Muy adecuado). En el segundo apartado se les pide que valoren en una escala de 1 a 4 (1: Nada, 2: Poco, 3: Bastante, 4: Mucho) los efectos de la aplicación del protocolo. En el último apartado los técnicos valoran en qué grado diferentes aspectos sirven como ayuda y facilitan la implementación 


\section{Figura 1}

Proceso del protocolo de valoración de situaciones de desprotección infantil.

Notificación de una posible situación de desprotección infantil

\section{INVESTIGACIÓN PREVIA:}

Planificación y ejecución (Trabajador/a social)

Valoración conjunta: evaluación del riesgo y las necesidades de intervención (todo el equipo)

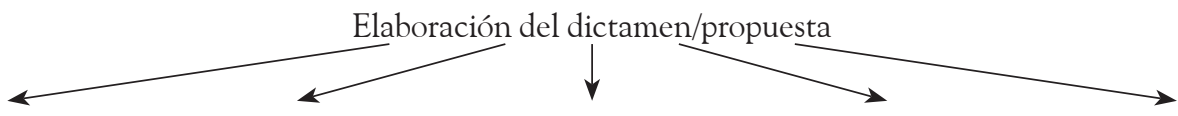
No hay intervención Acciones preventivas Desprotección: No hay información Desprotección no Da paso a la fase de suficiente (continúa compatible con la valoración la investigación) permanencia del me- $\downarrow \quad$ nor con la familia

\section{VALORACIÓN ESPECIALIZADA:}

Planificación conjunta del proceso de valoración (todo el equipo)

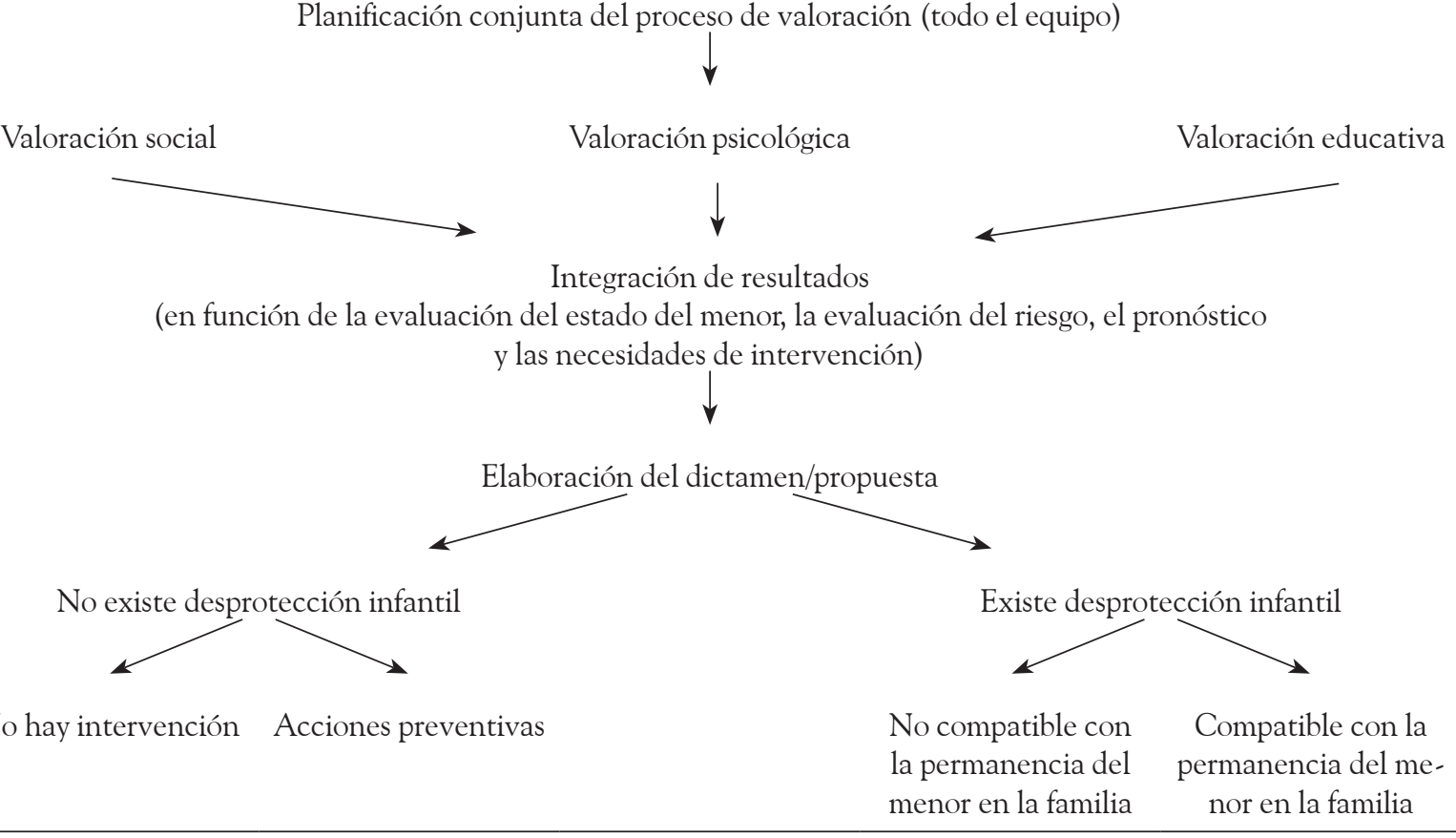

Fuente: elaboración propia.

del protocolo (escala de respuesta 1: Nada, 2: Poco, 3: Bastante, 4: Mucho).

\section{Procedimiento}

En el año 2005 se crea, en la Unidad de Infancia y Familia del IASS, el Programa de Prevención y Pro- moción, a través del cual se comienzan a desarrollar acciones en materia de promoción del bienestar infantil, prevención, asesoramiento técnico y jurídico, y planificación insular en el área de infancia y familia. Desde este organismo se establece la figura profesional del mediador de zona, que es un técnico especializado que sirve de enlace entre el progra- 
ma y los profesionales municipales. Asimismo, se configuran las mesas técnicas comarcales como espacio de trabajo compartido entre los mediadores de zona y los profesionales de los diferentes municipios. En el año 2006, y en este contexto de trabajo, se decide elaborar una propuesta técnica para la unificación insular de un protocolo de valoración de situaciones de desprotección infantil, cuyo objetivo era establecer, entre todas las administraciones implicadas, las condiciones básicas de investigación y valoración especializada de las situaciones de desprotección infantil, con unos criterios comunes para todos los profesionales. Durante el año 2007 los profesionales municipales comienzan a aplicar la versión inicial del protocolo contando con el asesoramiento y acompañamiento de los mediadores de zona. Paralelamente, se pone en marcha una comisión formada por técnicos de las diferentes administraciones implicadas (ayuntamientos, Unidad de Infancia y Dirección General de Protección del Menor y la Familia del Gobierno Autonómico) y por los autores de este trabajo, y que se encarga de realizar la evaluación de la implementación del protocolo.

Dentro de este proceso de evaluación, en el año 2008, los técnicos municipales que ya habían utilizado el protocolo cumplimentaron el cuestionario de evaluación para profesionales. Los cuestionarios fueron realizados de manera voluntaria, individual y anónima por los técnicos municipales, y los mediadores de zona se responsabilizaron de la entrega y recogida de los mismos.

\section{Análisis de datos}

Se analizaron las respuestas al cuestionario a través de un análisis de frecuencias, y se realizaron contrastes Chi-cuadrado para comprobar si existían diferencias en las respuestas en función de la participación o no en la elaboración del protocolo y en función del perfil profesional. Se utilizó el programa SPSS versión 15.

\section{Resultados}

\section{Valoración del contenido del Protocolo}

En la Figura 2 se puede observar que los profesionales, en porcentajes que van desde el $73.3 \%$ al $93.3 \%$, valoran como adecuados o muy adecuados los contenidos del protocolo para valorar situaciones de desprotección infantil. El porcentaje de acuerdo es especialmente alto al valorar el contenido relativo a la fase de IP (91.4\% adecuado + muy adecuado), el contenido relativo a la toma de decisiones en la fase de IP (93.3 \% adecuado + muy adecuado) y el contenido relativo al procedimiento de valoración ( $90.4 \%$ adecuado + muy adecuado). Se reduce ligeramente al valorar el modelo de planificación y registro de la información de la investigación previa (83.1\% adecuado + muy adecuado), el modelo de informe propuesto para la toma de decisiones ( $88.5 \%$ adecuado + muy adecuado) y el documento de planificación conjunta $(89.7 \%$ adecuado + muy adecuado). Y donde sí se reduce el porcentaje de acuerdo de forma significativa, es al valorar los modelos de informe de valoración conjunta (73.3\% adecuado + muy adecuado).

Es precisamente en esta última variable donde se detecta un contraste significativo entre la valoración realizada por parte de los psicólogos (solo el $52.4 \%$ realiza una valoración de adecuado + muy adecuado) en comparación con los trabajadores sociales y educadores ( $82.6 \%$ en ambos casos) $\left[\chi_{(4)}^{2}=9668, p<0.05\right]$.

No se detectan contrastes significativos entre las valoraciones realizadas por quienes participaron en la elaboración de protocolo y quienes no.

\section{Valoración de los efectos de la aplicación del protocolo}

En la Figura 3 se puede observar cómo la opinión de los profesionales sobre la contribución del protocolo varía de forma sustancial según el aspecto que se analiza.

En este sentido, se logra un alto acuerdo al valorar que el protocolo ha contribuido bastante o mucho a mejorar aspectos como: las condiciones 


\section{FIGURA 2}

Valoración del contenido para las diferentes fases del protocolo

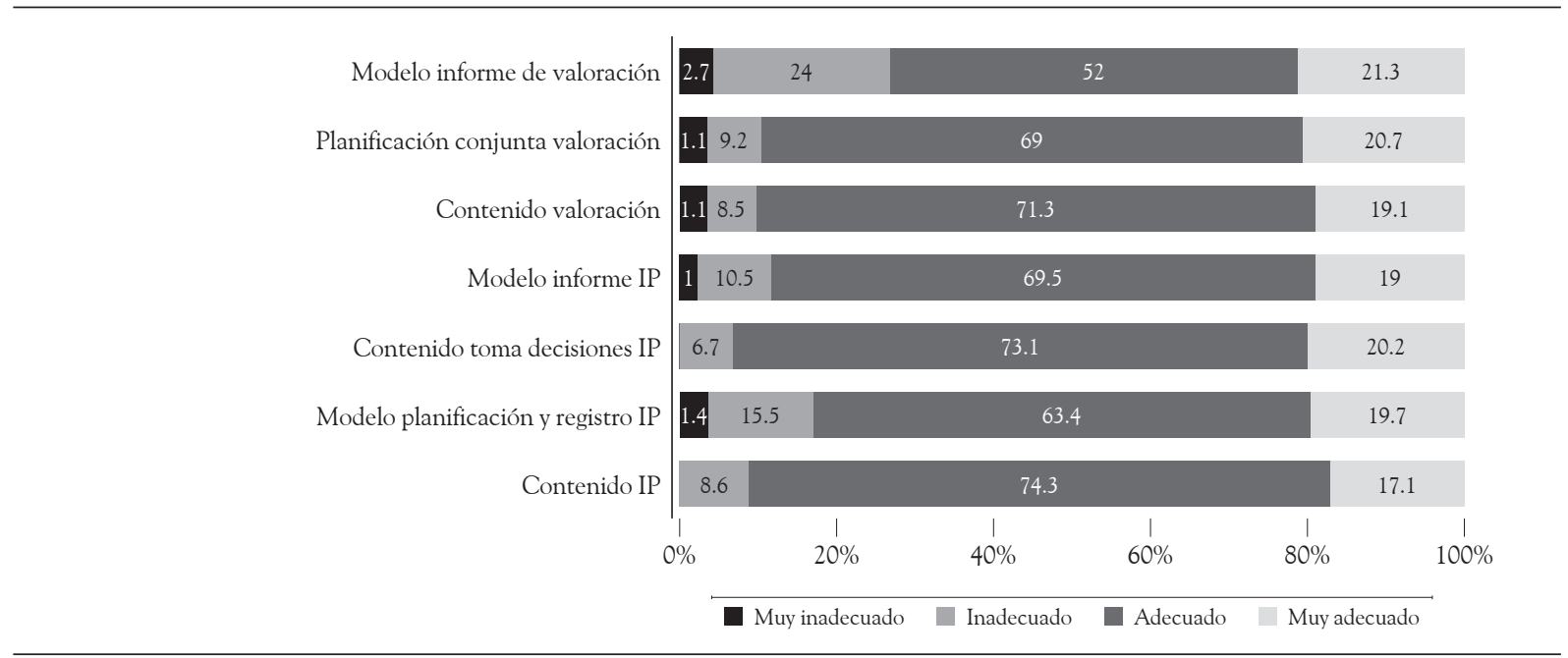

Fuente: elaboración propia.

Figura 3

Valoración de los efectos de la aplicación del protocolo

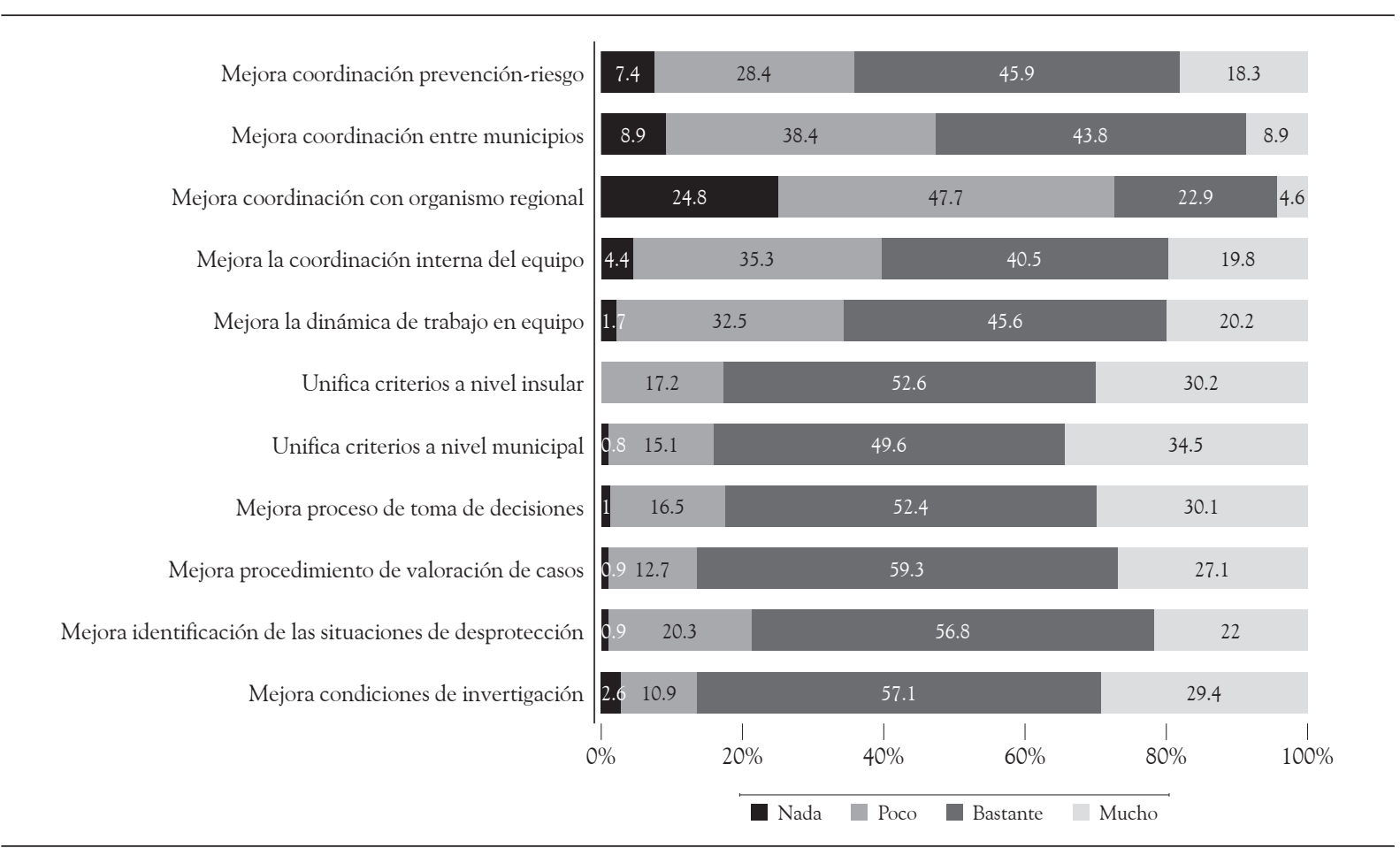

Fuente: elaboración propia.

básicas de la investigación (86.5\% bastante + mucho); el procedimiento para la valoración de los casos ( $86.4 \%$ bastante + mucho); el unificar criterios en cuanto a la valoración de la situación de desprotección infantil a nivel municipal (84.1\% bastante + mucho); el unificar criterios en cuanto a la valoración de la situación de desprotección infantil a nivel insular ( $82.8 \%$ bastante + mucho); 
la toma de decisiones (dictamen/propuesta) por parte del equipo ( $82.5 \%$ bastante + mucho) y la identificación de la existencia de desprotección infantil (78.8 \% bastante + mucho). Se observa más diversidad de opinión sobre si ha contribuido poco, bastante o mucho a mejorar aspectos como: la dinámica de trabajo en equipo $(65.8 \%$ bastante + mucho); la coordinación horizontal (equipos de prevención/equipos de riesgo) (64.2\% bastante + mucho); la coordinación interna del equipo (60.3\% bastante + mucho); y la coordinación horizontal (ayuntamiento - otros ayuntamientos (52.7\% bastante + mucho). El grado de acuerdo vuelve a ser alto, aunque en este caso en sentido contrario, al estimar el $72.5 \%$ de los profesionales que el protocolo ha contribuido nada (24.8\%) o poco $(47.7 \%)$ a mejorar la coordinación vertical (ayuntamiento - Dirección General de Protección del Menor y la Familia).

No se han detectado contrastes significativos en las opiniones de los profesionales ni en función de su participación o no en la elaboración del protocolo, ni en función de su perfil profesional.

\section{Valoración de los recursos en función de su ayuda en la implementación del protocolo}

En la Figura 4 se puede observar claramente cómo el asesoramiento del mediador, esto es, del profesional de la Unidad de Infancia y Familia que ha actuado como mediador entre la Unidad y los municipios, es el recurso en el que los profesionales expresan mayor acuerdo en que ha facilitado bastante o mucho la implementación del protocolo (96.5\% bastante + mucho). También se observa un amplio acuerdo en apreciar que ha facilitado bastante o mucho la implementación del protocolo: las mesas comarcales $(84.6 \%$ bastante + mucho) y la dinámica de trabajo del equipo (84.6\% bastante + mucho). Las opiniones son más diversas al valorar la contribución de resto de recursos: la coordinación ejercida por la persona responsable (coordinador/a de equipos, coordinador/a de SS.SS, coordinador/a de área...) (54.3\% bastante + mucho), la dotación de recursos personales en el municipio ( $51.3 \%$ bastante + mucho) y la dotación de recursos materiales en el municipio ( $47.7 \%$ bastante + mucho) .

En este apartado no se observan diferencias en la valoración realizada en función de si se ha participado o no en la elaboración, aunque sí en función del perfil profesional. Así, se detecta cómo los recursos materiales son peor valorados por los educadores (solo el $24.3 \%$ estiman que han facilitado bastante o mucho la implementación del protocolo), frente a los psicólogos (63.3 \%) y a los trabajadores sociales $(54.1 \%)\left[\chi_{(6)}^{2}=14.551\right.$, $p<0.05]$. Ocurre algo similar con los recursos personales que, en este caso, son mejor valorados por los psicólogos (71\%), frente a las valoraciones realizadas por trabajadores sociales (49\%) y por los educadores $(36.1 \%)\left[\chi_{(6)}^{2}=19.379, p<0.01\right]$.

\section{Discusión y conclusiones}

En el primer objetivo que nos planteamos en este trabajo pretendíamos conocer cómo valoran los técnicos municipales el protocolo. Los resultados muestran que el instrumento es valorado de manera positiva por los técnicos que lo utilizan, sobre todo en lo que se refiere al contenido conceptual y procedimental para desarrollar las tareas de investigación y valoración especializada. Aunque también reciben una buena valoración, se observa que es algo menor en el caso de los modelos de registro y de informes propuestos. Una posible explicación a estas pequeñas diferencias puede ser que los contenidos teóricos y procedimentales son los aceptados por la comunidad científica (Arruabarrena, 2002, 2009; Arruabarrena \& De Paúl, 1994; De Paúl, 2002; English et al., 2005; López, 1995, 2008; López et al., 1995; Manly, 2005; Muela, 2008; Observatorio de la Infancia, 2008; Rodrigo \& Máiquez, 2007) y, por lo tanto, ya conocidos por los profesionales, mientras que los modelos de registro y de informes son una novedad. Por otra parte, los modelos de registro y los informes requieren que el equipo se reúna y realice una puesta en común, lo que no es fácil en muchos casos, debido a la falta de tiempo o de cultura de trabajo en equipo, que son dos de las dificultades encontradas por los técnicos (Darlington et al., 2010; Martín \& Acie- 
FIGURA 4

Valoración de los recursos en función de su ayuda en la implementación del protocolo.

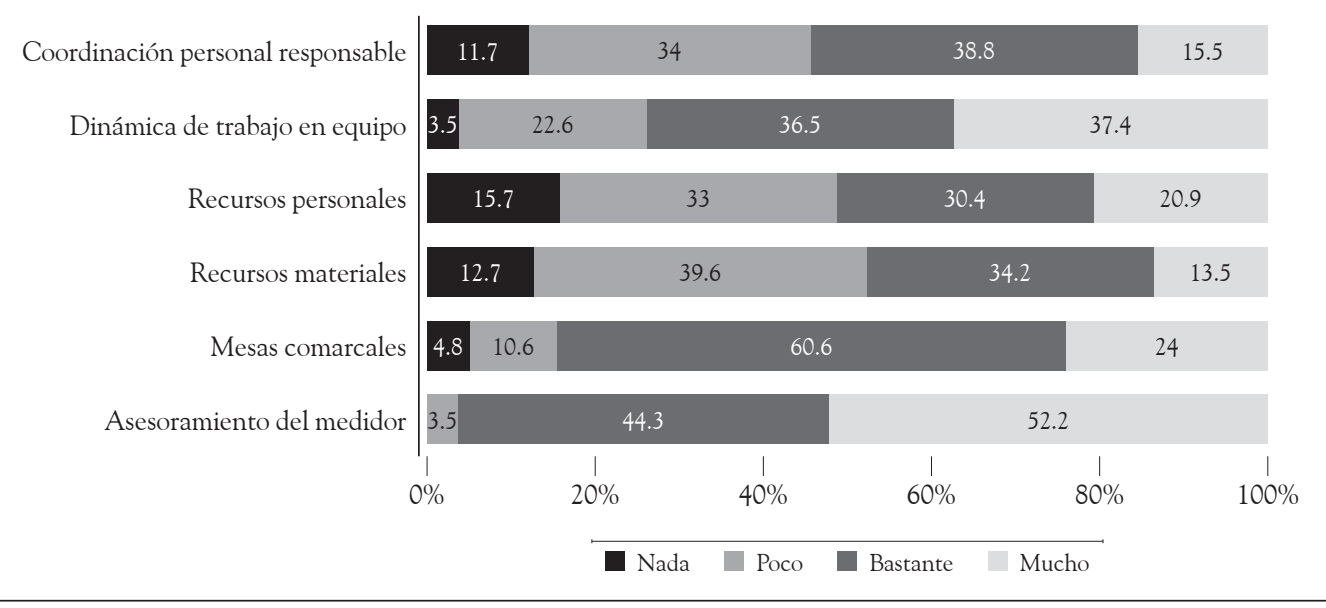

Fuente: elaboración propia.

go de Mendoza, 2010). Mención especial merece el hecho de que los psicólogos consideren peor el modelo de informe de valoración especializada, que los educadores y los trabajadores sociales. El desarrollo profesional de la Psicología en España ha llevado aparejada la proliferación de modelos de informes psicológicos adecuados a cada ámbito, incluido el de la protección infantil (Arruabarrena, 2009; Arruabarrena \& De Paúl, 1994), por lo que la obligación de hacer informes conjuntos con los demás perfiles supone un cambio de hábito que podría estar detrás de estas diferencias.

Con respecto al segundo de los objetivos de este trabajo, los resultados muestran que, según los profesionales, el protocolo mejora sustancialmente las condiciones para desarrollar las tareas de investigación y de valoración, mejorando las condiciones en las que se toman las decisiones. Además, consideran mayoritariamente que ayuda a unificar criterios, tanto a nivel municipal como insular. Se observa una mayor diversidad a la hora de valorar los efectos que la aplicación del protocolo ha tenido en aspectos como la dinámica de trabajo del equipo, la coordinación intra e inter equipos y la coordinación entre municipios. Probablemente, sean estos aspectos los que deben ayudar a la implementación del protocolo y no al revés, tal y como manifiestan muchos de los técnicos encuestados
(Martín \& Aciego de Mendoza, 2010). Lo mismo se puede decir del hecho de que la gran mayoría de los profesionales considera que el protocolo no ayuda a mejorar la coordinación vertical, en este caso con la Dirección General de Protección del Menor y la Familia, que es el órgano competente en materia de protección infantil a nivel autonómico. Como era lógico esperar, un instrumento que se crea en un proceso participativo a nivel horizontal, difícilmente puede tener incidencia en la coordinación vertical, cuya mejora depende de otros aspectos (De Paúl, 2009).

El tercer objetivo de este estudio pretendía conocer cuáles son los recursos que facilitan la implementación del protocolo. Los resultados muestran que, según la opinión de los técnicos municipales, la figura del mediador de zona y las mesas comarcales son los recursos que más ayudan a la implementación del protocolo. El papel de estos dos recursos a la hora de dar a conocer el instrumento y su filosofía, además de generar espacios para compartir y generar un conocimiento y un lenguaje común, los convierten en recursos claves a la hora de implementar, con garantías de éxito, nuevos instrumentos y procedimientos de trabajo compartidos, y evitar que se desarrollen dinámicas diferentes en cada equipo (Casas \& Montserrat, 2002). Por otro lado, la dinámica 
de trabajo en equipo también es valorada como un recurso importante. Lógicamente, la filosofía del protocolo está basada en el trabajo en equipo, en el que los diferentes profesionales implicados deben actuar de manera coordinada, por lo que el establecimiento de dinámicas adecuadas es, en este sentido, una pieza clave.

Mayor diversidad se observa a la hora de valorar el papel de la coordinación ejercida por la persona responsable, y de los recursos personales y materiales. Esto posiblemente sea debido a las diferencias observadas en los diferentes municipios, en cuanto a los recursos con los que cuentan y a las dinámicas que ejercen las diferentes personas encargadas de las tareas de coordinación (Martín \& Aciego de Mendoza, 2010). Las diferencias observadas en función del perfil profesional también merecen un comentario. Los psicólogos valoran más la ayuda de los recursos personales, sobre todo en comparación con los educadores. Este resultado podría ser explicado por el hecho de que en aquellos equipos en los que hay psicólogos, suelen estar presentes los otros perfiles, existiendo un número de técnicos que permite el trabajo en equipo. En cambio, en algunos municipios, sobre todo en los más pequeños, es más complicado, desde un punto de vista económico, contratar titulados superiores, siendo además pocos los técnicos que forman el equipo y que, además, pueden trabajar en lugares diferentes debido a la dispersión poblacional que existe en algunos municipios rurales.

Otra diferencia en función del perfil es la peor valoración de los recursos materiales que los educadores hacen, en comparación con psicólogos y trabajadores sociales. La definición del perfil profesional del educador todavía es problemática, sobre todo si la comparamos con la de los psicólogos y los trabajadores sociales. Estos tienen mejor definidas sus funciones y sus instrumentos (recursos materiales), mientras que los educadores manifiestan que no tienen claro su papel dentro de los equipos multidisciplinares, ni los instrumentos que deben utilizar (Martín \& Aciego de Mendoza, 2010). Hay que decir que en la universidad más cercana, la de La Laguna, no se imparte el título de educador social, que sería la titulación de referencia para los educadores. Esto hace que se acceda al puesto de educador con formación muy diferente: psicólogos, pedagogos, trabajadores sociales, maestros, etc., lo que dificulta aún más la definición del perfil profesional.

Un último resultado destacable es que no se han encontrado diferencias en la valoración que los profesionales hacen del protocolo, en función de si han participado o no en su elaboración. Esto puede ser interpretado como un indicador de bondad del propio instrumento, ya que aquellos que no participaron en su elaboración lo valoran igual, es decir positivamente, que aquellos que tuvieron protagonismo en su elaboración.

En definitiva, los resultados de este trabajo nos indican que los profesionales encargados de utilizar, en su práctica diaria, el protocolo lo valoran de manera positiva, lo que podría interpretarse como un indicador de éxito en su implementación, más allá de la propia bondad y validez del propio instrumento (Hernández, 1993). Además, los datos aportados en este trabajo sugieren que la elaboración de instrumentos de este tipo, desde un enfoque participativo en el que tengan protagonismo los técnicos municipales, acompañados de figuras como la de los mediadores de zona que apoyen en el proceso de implementación, mejora la labor que se desarrolla desde los servicios sociales municipales, se unifican los criterios y el lenguaje, y se mejora la coordinación horizontal, aunque no la vertical (De Paúl, 2009). No obstante, la enorme diversidad que se observa en los diferentes municipios en cuanto a recursos, tanto personales como materiales, como a las dinámicas de coordinación, parecen mediatizar la implementación del protocolo. Por otro lado, es conveniente tener en cuenta los diferentes perfiles profesionales que configuran los equipos, sobre todo en el caso de los educadores, cuya indefinición profesional parece dificultar su labor y la del resto del equipo, ya que se corre el riesgo de que se actúe más en función de la formación previa que del propio rol profesional.

No queremos finalizar sin comentar cuál es la principal limitación de este trabajo. Aunque desde un punto de vista práctico la valoración que realizan los profesionales nos aporta una información 
valiosa sobre el protocolo, es necesario realizar otros estudios sobre su validez y fiabilidad como instrumento, tanto a través del seguimiento de la evolución de los casos como de la realización de análisis de fiabilidad interjueces.

\section{Referencias}

Arad-Davidzon, B. \& Benbenishty, R. (2008). The role of workers' attitudes and parent and child wishes in child protection workers' assessment and recommendation regarding removal and reunification. Children and Youth Services Review, 30, 107-121.

Arruabarrena, M. I. (2002). Investigación en situaciones de desprotección infantil. En J. de Paúl \& M. I. Arruabarrena (Eds.), Manual de Protección Infantil (pp. 181-228). Barcelona: Masson.

Arruabarrena, M. I. (2009). Procedimiento y criterios para la evaluación y la intervención con familias y menores en el ámbito de la protección infantil. Papeles del Psicólogo, 30, 13-23.

Arruabarrera, M. I. \& De Paúl, J. (1994). Maltrato a los niños en la familia. Evaluación y tratamiento. Madrid: Pirámide.

Britner, P. A. \& Mossler, D. G. (2002). Professionals' decision-making about out-of-home placements following instances of child abuse. Child Abuse and Neglect, 26, 317-332.

Casas, F. \& Montserrat, C. (2002). Interdisciplinary teams and decision making in child protection systems: The experience of Catalonia. International Journal of Child and Family Welfare, 5, 146-158.

Consejo General de Colegios Oficiales de Psicólogos. (Ed.). (2009). Intervención psicosocial en protección a la infancia [Número monográfico]. Papeles del Psicólogo, 30 (1).

Daniel, B., Vincent, S., Farrall, E. \& Arney, F. (2009). How is the concept of resilience operationalised in practice with vulnerable children? International Journal of Child and Family Welfare, 12, 2-21.

Darlington, Y., Healy, K. \& Feeney, J. A. (2010). Approaches to assessment and intervention across four types of child and family welfare services. Children and Youth Services Review, 32, 356 364.
De Paúl, J. (2002). Diferentes situaciones de desprotección infantil. En J. de Paúl \& M. I. Arruabarrena (Eds.), Manual de Protección Infantil (pp. 3-23). Barcelona: Masson.

De Paúl, J. (2009). La intervención psicosocial en protección infantil en España: evolución y perspectivas. Papeles del Psicólogo, 30, 4-12.

English, D. J., Bangdiwala, S. I. \& Runyan, D. K. (2005). The dimensions of maltreatment: Introduction. Child Abuse and Neglect, 29, 441-460.

Fallon, B., Trocmé, N., Fluke, J., Maclaurin, B., Tonmyr, L. \& Yuan, Y. Y. (2010). Methodological challenges in measuring child maltreatment. Child Abuse and Neglect, 34, 70-79.

González, O. (2002). Regulación jurídica de la protección infantil. En J. de Paúl \& M. I. Arruabarrena (Eds.), Manual de Protección Infantil (pp. 65-139). Barcelona: Masson.

Hernández, P. (1993). Programa y metaprograma en la intervención psicológica. En J. A. Beltrán, V. Bermejo, M. D. Prieto \& D. Vence (Eds.), Intervención psicopedagógica (pp. 400-417). Madrid: Pirámide.

Instituto Insular de Atención Social y Sociosanitaria. (2008). Protocolo de valoración de situaciones de desprotección infantil en la isla de Tenerife. S/C de Tenerife: IASS.

Jent, J. F., Merrick, M. T., Dandes, S. K., Lambert, W. F., Haney, M. L. \& Cano, N. M. (2009). Multidisciplinary assessment of child maltreatment: A multi-site pilot descriptive analysis of the Florida Child Protection Team model. Children and Youth Services Review, 31, 896-902.

Lázaro, S. (2009). Resiliencia en niños y adolescentes: revisión teórica e implicaciones para la intervención psicoeducativa en situaciones de maltrato familiar. Estudios de Psicología, 30, 89-104.

López, F. (1995). Necesidades de la infancia y protección infantil (Vol. 1). Madrid: Ministerio de Trabajo y Asuntos Sociales.

López, F. (2008). Necesidades en la infancia y en la adolescencia. Respuesta familiar, escolar y social. Madrid: Pirámide.

López, F., Torres, B., Fuertes, Z., Sánchez, J. M. \& Merino, J. (1995). Necesidades de la infancia y protección infantil (Vol. 2). Madrid: Ministerio de Trabajo y Asuntos Sociales. 
Manly, J. T. (2005). Advances in research definitions of child maltreatment. Child Abuse and Neglect, 29, 425-439.

Martín, E. \& Aciego de Mendoza, R. (2010). Evaluación cualitativa de la implementación de un protocolo de valoración de situaciones de desprotección infantil. Manuscrito en preparación.

Martín, J. (2005). La intervención ante el maltrato infantil. Una revisión del sistema de protección. Madrid: Pirámide.

Martín, J. (2009). Protección de menores: una institución en crisis. Madrid: Pirámide.

Montero, I. \& León, O. G. (2007). A guide for naming research studies in Psychology. International Journal of Clinical and Health Psychology, 7, 847-862.

Muela, A. (2008). Hacia un sistema de clasificación nosológico del maltrato infantil. Anales de Psicología, 24, 77-87.

Observatorio de la Infancia. (2008). Protocolo básico de intervención contra el maltrato infantil. Madrid: Ministerio de Trabajo y Asuntos Sociales.

Ramos-Álvarez, M. M., Moreno-Fernández, M. M., Valdés-Conroy, B. \& Catena, A. (2008). Criteria of the peer-review process for publication of experimental and quasiexperimental research in Psychology: A guide for creating research papers. International Journal of Clinical and Health Psycho$\log y, 8,751-764$.
Rodrigo, M. J. \& Máiquez, M. L. (2007). Manual de buenas prácticas de los Servicios Sociales municipales dirigidos al menor y la familia. S/C de Tenerife: Consejería de Empleo/Asuntos Sociales del Gobierno de Canarias.

Rodrigo, M. J., Camacho, J., Máiquez, M. L., Byrne, S. $\&$ Benito, J. M. (2009). Factores que influyen en el pronóstico de recuperación de las familias en riesgo psicosocial: el papel de la resiliencia del menor. Psicothema, 21, 90-96.

Rodrigo, M. J., Máiquez, M. L., Martín, J. C. \& Byrne, S. (2008). Preservación familiar. Un enfoque positivo para la intervención con las familias. Madrid: Pirámide.

Rodríguez, G., Camacho, J., Rodrigo, M. J., Martín, J. C. \& Máiquez, M. L. (2006). Evaluación del riesgo psicosocial en familias usuarias de los Servicios Sociales Municipales. Psicothema, 18, 200-206.

Runyan, D. K., Cox, C. E., Dubowitz, H., Newton, R. R., Upadhyaya, M., Kotch, J. B. et al. (2005). Describing maltreatment: Do child protective service report and research definitions agree? Child Abuse and Neglect, 29, 461-477.

Shlonsky, A. \& Wagner, D. (2005). The next step: Integrating actuarial risk assessment and clinical judgment into an evidence-based practice framework in CPS case management. Children and Youth Services Review, 27, 409-427. 